Quim. Nova, Vol. 33, No. 1, 52-56, 2010

\title{
TERMOCROMISMO EM SOLUÇÕES DE ALCÓXIDOS DE VANÁDIO(IV): UMA ABORDAGEM PELA MODELAGEM MOLECULAR
}

\author{
Alexandre C. de Freitas, Kátia C. M. Westrup, Giovana G. Nunes, Denis J. Gulin, Roberto L. A. Haiduke, Jaísa F. Soares \\ e Eduardo L. de Sá* \\ Departamento de Química, Setor de Ciências Exatas, Universidade Federal do Paraná, CP 19081, 81530-900 Curitiba - PR, Brasil
}

Recebido em 2/12/08; aceito em 12/7/09; publicado na web em 27/11/09

\begin{abstract}
THERMOCHROMIC BEHAVIOR OF VANADIUM(IV) ALKOXIDES IN SOLUTION: A MOLECULAR MODELING APROACH. The thermochromic behavior exhibited by vanadium(IV) alkoxides, $\left[\mathrm{V}_{2}\left(\mu-\mathrm{OPr}^{\mathrm{i}}\right)_{2}\left(\mathrm{OPr}^{\mathrm{i}}\right)_{6}\right]$ and $\left[\mathrm{V}_{2}(\mu-\mathrm{ONep})_{2}(\mathrm{ONep})_{6}\right]$, $\mathrm{OPr}^{\mathrm{i}}=$ isopropoxide and ONep $=$ neopentoxide, was studied by molecular modeling using DFT, TDDFT and INDO/S methods. The vibrational and electronic spectra calculated for $\left[\mathrm{V}_{2}\left(\mu-\mathrm{OPr}^{\mathrm{i}}\right)_{2}\left(\mathrm{OPr}^{\mathrm{i}}\right)_{6}\right]$ were very similar to the experimental data registered for crystalline samples of the complex and for its solutions at low temperature $(<210 \mathrm{~K})$, while spectra recorded at high temperature $(>$ $315 \mathrm{~K})$ were compatible with those calculated for the monomeric form, $\left[\mathrm{V}\left(\mathrm{OPr}_{4}\right)_{4}\right]$. These results consistently point to a monomer/ dimer equilibrium as an explanation for the solution thermochromism of $\left\{\mathrm{V}\left(\mathrm{OPr}^{\mathrm{i}}\right)_{4}\right\}_{\mathrm{n}}$. In spite of the structural similarity between $\left[\mathrm{V}_{2}(\mu-\mathrm{ONep})_{2}(\mathrm{ONep})_{6}\right]$ and $\left[\mathrm{V}_{2}\left(\mu-\mathrm{OPr}_{2}\right)_{2}\left(\mathrm{OPr}^{\mathrm{i}}\right)_{6}\right]$ in the solid state, the thermochromic behavior of the former could not be explained by the same model, and the possibility of tetranuclear aggregation at low temperatures was also investigated.
\end{abstract}

Keywords: thermochromism; vanadium(IV) alkoxides; molecular modeling.

\section{INTRODUÇÃO}

Quando a variação de temperatura provoca mudanças estruturais em nível microscópico, quer sejam elas drásticas como alterações conformacionais, ou sutis como na variação do estado de spin, temos o fenômeno de termocromismo. Assim como em todo equilíbrio químico, processos reversíveis que envolvem a agregação e a dissociação de moléculas - que podem explicar o comportamento termocrômico de um sistema - são dependentes da temperatura. Obviamente, para que estes processos impliquem em termocromismo, as formas agregadas e dissociadas devem exibir diferenças marcantes nos espectros de absorção ou emissão de fótons.

Como a temperatura não é uma variável que aparece na Equação de Schrödinger, não há uma correlação direta entre o uso da mecânica quântica e da modelagem molecular como ferramentas de interpretação do comportamento termocrômico de um sistema. Quando a realização de medidas espectroscópicas com temperatura variável não é possível, a proposição da natureza das espécies químicas envolvidas e do mecanismo pelo qual o termocromismo opera deve ser embasada no senso químico do investigador. Apesar de não ser um método direto ou que conduza necessariamente a conclusões definitivas, a abordagem do termocromismo pela modelagem molecular permite que o investigador proponha várias estruturas e mecanismos, teste-os através do confronto com resultados experimentais ou mesmo sugira outras explicações para o fenômeno.

Os alcóxidos homolépticos de metais de transição têm sido empregados como precursores de óxidos metálicos de grande aplicação tecnológica. ${ }^{1}$ Este uso pode ser explicado pela facilidade com que óxidos de alta pureza e de estequiometria e estrutura definidas podem ser obtidos a partir destes alcóxidos, principalmente através do processo sol-gel ou pelos métodos de deposição química ou física de vapor (CVD e PVD) ${ }^{1,2}$ As características dos produtos advindos destes processos são amplamente dependentes das estruturas moleculares dos precursores em solução.

Acredita-se que muitos alcóxidos de metais de transição (bloco d) sejam protagonistas de equilíbrios de agregação e dissociação

\footnotetext{
*e-mail: edulsa@quimica.ufpr.br
}

entre as formas monomérica e dimérica, ${ }^{3}$ comportamento que pode ser relacionado com a facilidade de formação de ligações $M-(\mu-$ OR)-MOR, com alcóxidos em pontes $\mu_{2}$ ou $\mu_{3}$. Já na química de alcóxidos homolépticos de metais do bloco f, a oligomerização é um fenômeno comum, pois o grande raio iônico do centro de coordenação permite que o número de ligantes ao seu redor seja elevado. Apesar destes precedentes, o sistema em estudo no presente trabalho é o primeiro em que o fenômeno da oligomerização é acompanhado de termocromismo.

O alcóxido de vanádio(IV) formado em presença de íons isopropóxido é conhecido desde 1962 quando foi sintetizado por Bradley, que inicialmente acreditava se tratar de $\left[\mathrm{V}\left(\mathrm{OPr}^{\mathrm{i}}\right)_{4}\right]$, onde $\mathrm{OPr}^{\mathrm{i}}=$ isoproprila. ${ }^{4}$ Entretanto, a determinação da massa molar do produto por crioscopia em benzeno mostrou que se tratava de uma estrutura de agregação com um grau de associação de 1,17. Hoje sabe-se que, em fase cristalina, se tem um composto dimérico de fórmula $\left[\mathrm{V}_{2}\left(\mu-\mathrm{OPr}^{\mathrm{i}}\right)_{2}\left(\mathrm{OPr}^{\mathrm{i}}\right)_{6}\right] .{ }^{5}$ Recentemente, utilizando uma outra rota sintética, $\left[\mathrm{V}_{2}\left(\mu-\mathrm{OPr}^{\mathrm{i}}\right)_{2}\left(\mathrm{OPr}^{\mathrm{i}}\right)_{6}\right]$ foi obtido em forma de monocristais, ${ }^{6}$ que, dissolvidos em tolueno ou hexano, apresentaram um comportamento termocrômico marcante, com colorações que variavam do amareloouro (abaixo de $210 \mathrm{~K}$ ) ao azul-cobalto (a partir de $315 \mathrm{~K}$ ), passando pelo verde nas temperaturas intermediárias. ${ }^{7} \mathrm{Um}$ comportamento termocrômico semelhante foi observado para um outro alcóxido de vanádio(IV), o $\left[\mathrm{V}_{2}(\mu-\mathrm{ONep})_{2}(\mathrm{ONep})_{6}\right]$, cuja estrutura cristalina é análoga à do $\left[\mathrm{V}_{2}\left(\mu-\mathrm{OPr}^{\mathrm{i}}\right)_{2}\left(\mathrm{OPr}^{\mathrm{i}}\right)_{6}\right] .{ }^{8}$ Entretanto, em especial no sistema com neopentóxido, não há evidências espectroscópicas inequívocas sobre a natureza das espécies moleculares que mudam a composição do sistema em resposta à variação da temperatura.

Este trabalho propôs-se a sugerir a natureza das espécies químicas responsáveis pelo comportamento termocrômico tão incisivo destes alcóxidos de vanádio(IV), $\left[\mathrm{V}_{2}(\mu-\mathrm{OR})_{2}(\mathrm{OR})_{6}\right], \mathrm{R}=$ isopropila ou neopentila. Para que este objetivo fosse alcançado, as ferramentas de modelagem molecular, com ênfase no emprego da Teoria do Funcional de Densidade (DFT, Density Functional Theory), da Teoria do Funcional de Densidade Dependente do Tempo (TDDFT, Time Dependent Density Functional Theory) e da parametrização semiempírica INDO/S, foram utilizadas. 


\section{PARTE EXPERIMENTAL}

Todas as operações relativas à síntese e à caracterização dos complexos foram realizadas sob atmosfera de $\mathrm{N}_{2}$ (pureza 99,999\%, White Martins) ou sob vácuo de $10^{-3}-10^{-4}$ Torr, utilizando técnicas de Schlenk ou glove-box. Os solventes foram purificados por métodos padronizados, ${ }^{9}$ secados exaustivamente e destilados antes do uso. Cristais dos alcóxidos de vanádio $\left[\mathrm{V}_{2}(\mu \text {-ONep })_{2}(\mathrm{ONep})_{6}\right]$ e $\left[\mathrm{V}_{2}\left(\mu-\mathrm{OPr}^{\mathrm{i}}\right)_{2}\left(\mathrm{OPr}^{\mathrm{i}}\right)_{6}\right]$ foram obtidos em rendimentos de 40 e $60 \%$, respectivamente, por uma rota sintética simples e reprodutível. ${ }^{8}$ Os espectros de absorção na região do infravermelho (400 a $4000 \mathrm{~cm}^{-1}$ ) com transformada de Fourier (IV-TF) foram obtidos em um instrumento BIO-RAD, Excalibur Series (FTS 3500 GX). Os espectros foram registrados em soluções com hexano ou em emulsões das amostras pulverizadas em óleo mineral (Nujol). Os espectros eletrônicos (UV-Vis, 190 a 900 nm) foram obtidos em um instrumento Shimadzu UV-2401 PC, em soluções com hexano, tolueno ou a partir de emulsões em óleo mineral.

\section{MÉTODOS COMPUTACIONAIS}

Os complexos de vanádio(IV) com grupos isopropóxido e neopentóxido, em fase sólida cristalina, apresentam-se sob a forma de dímeros cujas fórmulas moleculares são, respectivamente, $\left[\mathrm{V}_{2}\left(\mu-\mathrm{OPr}^{\mathrm{i}}\right)_{2}\left(\mathrm{OPr}^{\mathrm{i}}\right)_{6}\right]$ e $\left[\mathrm{V}_{2}(\mu-\mathrm{ONep})_{2}(\mathrm{ONep})_{6}\right]$. As estruturas destas espécies foram determinadas por difratometria de raios $\mathrm{X}$ e estão mostradas nas Figuras 1 e 2. ${ }^{6,8}$ Estes resultados cristalográficos serviram de ponto de partida para a otimização das geometrias das formas diméricas em solução com tolueno. A inclusão do solvente foi feita pelo emprego do método PCM (Polarizable Continuum Model). ${ }^{10}$ As geometrias das formas monoméricas foram geradas a partir das estruturas cristalográficas das formas diméricas, através da eliminação de átomos escolhidos com base em critérios de simetria molecular. Os cálculos foram feitos inicialmente utilizando-se a aproximação HF/6$31 \mathrm{G}^{11}$ disponível no pacote de programas GAMESS, ${ }^{12}$ e depois no nível B3LYP/LANL2DZ, ${ }^{13}$ disponível no pacote Gaussian03, ${ }^{14}$ ambos disponibilizados no Laboratório Central de Processamento de Alto Desempenho da Universidade Federal do Paraná (LCPAD/UFPR). A partir das estruturas otimizadas, os espectros vibracionais foram calculados utilizando a metodologia DFT com a mesma função de base empregada na otimização da geometria. As estruturas moleculares constituíram-se como mínimos locais, o que pôde ser caracterizado pela ausência de frequências vibracionais negativas nos espectros calculados. Os espectros eletrônicos foram obtidos utilizando-se as metodologias TDDFT e ZINDO, ${ }^{15,16}$ implementadas no programa Gaussian03, empregando-se 30 estados excitados como espaço ativo.

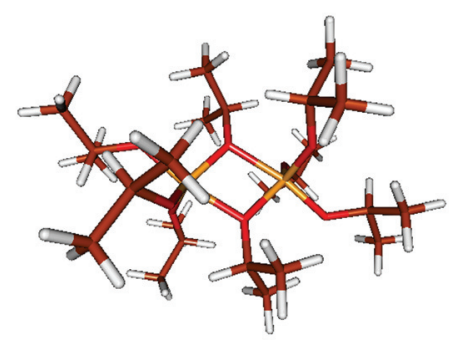

Figura 1. Representação da estrutura em estado sólido da espécie $\left[V_{2}\left(\mu-O P r^{i}\right)_{2}\left(\mathrm{OPr}^{i}\right)_{6}\right], \mathrm{OPr}^{i}=$ isopropila

\section{RESULTADOS E DISCUSSÃO}

Os espectros eletrônicos e vibracionais calculados não se alteraram sensivelmente com a inclusão do solvente utilizando-se o método

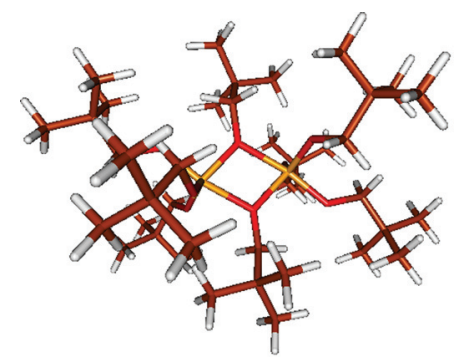

Figura 2. Representação da estrutura em estado sólido da espécie $\left[\mathrm{V}_{2}(\mu \text {-ONep })_{2}(\mathrm{ONep})_{6}\right]$, ONep = neopentila

PCM. Este resultado é congruente com aquele obtido por Nemykin e colaboradores no estudo dos fatores que influenciam as energias de excitação vertical de ftalocianinas. ${ }^{17}$ Desta forma, nas discussões que se seguem não serão feitas distinções entre resultados obtidos considerando-se ou não a presença do solvente.

\section{Sistema $\left[\mathrm{V}_{2}\left(\mu-\mathrm{OPr}^{\mathrm{i}}\right)_{2}\left(\mathrm{OPr}^{\mathrm{i}}\right)_{6}\right] /\left[\mathrm{V}\left(\mathrm{OPr}^{\mathrm{i}}\right)_{4}\right]$}

Para o composto binuclear, $\left[\mathrm{V}_{2}\left(\mu-\mathrm{OPr}^{\mathrm{i}}\right)_{2}\left(\mathrm{OPr}^{\mathrm{i}}\right)_{6}\right]$, a geometria de partida foi a estrutura determinada por difratometria de raios $\mathrm{X}$, com multiplicidade de spin $(2 \mathrm{~S}+1)$ igual a 3 . Após convergência, a geometria otimizada deste dímero manteve uma precisão, em relação aos dados experimentais, de cinco casas decimais para comprimentos de ligação e de três casas para ângulos de ligação.

Para a espécie monomérica, $\left[\mathrm{V}\left(\mathrm{OPr}^{\mathrm{i}}\right)_{4}\right]$, uma estrutura gerada a partir de comprimentos e ângulos de ligação padrão, com multiplicidade de spin do estado fundamental igual a 2 , foi o ponto inicial do cálculo. Semelhantemente ao descrito por Haaland et al. para a estrutura molecular do complexo $\left[\mathrm{V}\left(\mathrm{OBu}^{\mathrm{t}}\right)_{4}\right]\left(\mathrm{Bu}^{\mathrm{t}}=\right.$ terc -butila $)$ determinada em fase gasosa por difração de elétrons, ${ }^{18} \mathrm{o}$ poliedro de coordenação calculado para o $\left[\mathrm{V}\left(\mathrm{OPr}^{\mathrm{i}}\right)_{4}\right]$ apresenta-se ligeiramente achatado em relação a um tetraedro perfeito, adotando a simetria $S_{4}$. Os ângulos médios de ligação $(\mathrm{O}-\mathrm{V}-\mathrm{O})$ calculados para o [ $\left.\mathrm{V}\left(\mathrm{OPr}^{\mathrm{i}}\right)_{4}\right]$ são $112,2(16)^{\circ}$ e $108,4(2)^{\circ}$, comparados com $115,1(19)^{\circ}$ e $106,7(11)^{\circ}$ no $\left[\mathrm{V}\left(\mathrm{OBu}^{\mathrm{t}}\right)_{4}\right]$, o que indica uma distorção menor no complexo com isopropóxido. Esta distorção pode ser atribuída a uma combinação dos efeitos $(i)$ da doação $\pi$ do alcóxido para os orbitais $\mathrm{d}_{\mathrm{x}-\mathrm{y}}^{2}{ }^{2} \mathrm{e} / \mathrm{ou} \mathrm{d}_{\mathrm{z}}{ }^{2} \mathrm{do}$ vanádio(IV) e (ii) da repulsão espacial entre os ligantes volumosos. ${ }^{18}$

$\mathrm{O}$ espectro eletrônico experimental de uma solução de $\left[\mathrm{V}_{2}\left(\mu-\mathrm{OPr}^{\mathrm{i}}\right)_{2}\left(\mathrm{OPr}^{\mathrm{i}}\right)_{6}\right]$ em tolueno apresenta uma mudança nítida e reversível com a variação da temperatura (Figura 3). Nota-se claramente a presença de uma banda em $674 \mathrm{~nm}$ em temperaturas altas, que diminui em intensidade com o resfriamento do sistema; concomitantemente, observa-se o aparecimento de uma banda em $470 \mathrm{~nm}$. O ponto isosbéstico em $530 \mathrm{~nm}$ sugere a presença de somente duas espécies químicas em equilíbrio na solução (formas azul e amarela); a cor verde resultaria da coexistência destas formas em temperaturas intermediárias. A espécie formada a altas temperaturas seria o complexo mononuclear $\left[\mathrm{V}\left(\mathrm{OPr}_{4}\right)_{4}\right]$, a partir do qual se cristaliza o $\left[\mathrm{V}_{2}\left(\mu-\mathrm{OPr}^{\mathrm{i}}\right)_{2}\left(\mathrm{OPr}^{\mathrm{i}}\right)_{6}\right]$. A espécie predominante em baixas temperaturas seria uma molécula binuclear, como a da estrutura cristalina. Ocorreria assim um equilíbrio de agregação monômero-dímero. A Tabela 1 mostra os valores dos sinais experimentais e dos calculados para estas duas espécies em solução.

A análise da Tabela 1 mostra que a proposta mecanística feita no parágrafo anterior é condizente com os resultados experimentais. No caso da espécie monomérica, são previstos três sinais, com um máximo em $678 \mathrm{~nm}$ calculado por TDDFT (B3LYP/LANL2DZ). Experimentalmente, há somente um sinal bastante alargado com máximo em $674 \mathrm{~nm}$, que pode perfeitamente estar ocultando os sinais de menor intensidade previstos pelo cálculo TDDFT. Para a espécie 
supostamente presente à baixa temperatura, há a previsão da banda observada experimentalmente em $470 \mathrm{~nm}$ por ambos os métodos teóricos usados. É também comum aos dois métodos teóricos a previsão, para a espécie de baixa temperatura, de uma banda na região de menor energia, entre 700 e $800 \mathrm{~nm}$, também detectada experimentalmente (Figura 3). Assim, os resultados INDO/S e TDDFT reforçam-se mutuamente e apontam para uma confirmação da proposta de agregação.

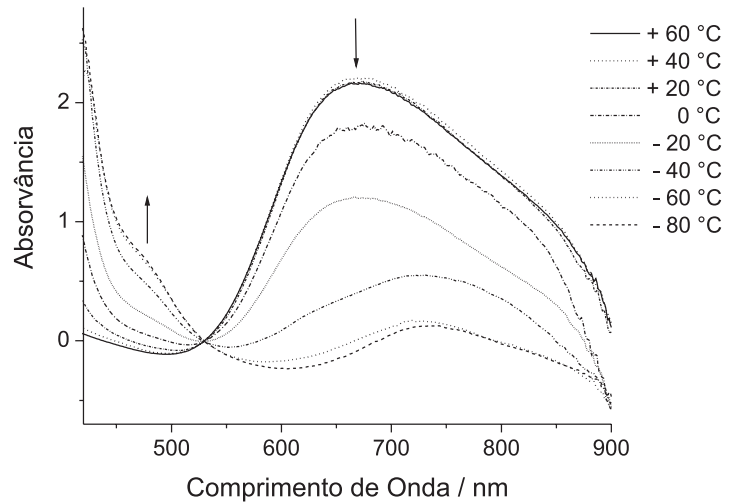

Figura 3. Espectro eletrônico da solução de $\left[\mathrm{V}_{2}\left(\mu-\mathrm{OPr}^{i}\right)_{2}\left(\mathrm{OPr}^{i}\right)_{6}\right]$, concentração $1,84 \times 10^{-2}$ mol $L^{-1}$ em tolueno, em temperaturas de $-80 a+60{ }^{\circ} \mathrm{C}$. As setas indicam o sentido da variação de absorvância em resposta à diminuição da temperatura

Tabela 1. Comparação entre os sinais calculados e os experimentais para as espécies monomérica $\left(\left[\mathrm{V}\left(\mathrm{OPr}^{\mathrm{i}}\right)_{4}\right]\right)$ e dimérica $\left(\left[\mathrm{V}_{2}\left(\mu-\mathrm{OPr}^{\mathrm{i}}\right)_{2}\left(\mathrm{OPr}^{\mathrm{i}}\right)_{6}\right]\right)$ tentativamente atribuídas como espécies de alta e de baixa temperaturas, respectivamente

\begin{tabular}{lcc}
\hline \multirow{2}{*}{ Metodologia } & \multicolumn{2}{c}{ Transição $(\lambda / \mathrm{nm})^{*}$} \\
& {$\left[\mathrm{~V}\left(\mathrm{OPr}^{\mathrm{i}}\right)_{4}\right]$} & {$\left[\mathrm{V}_{2}\left(\mu-\mathrm{OPr}_{2}^{\mathrm{i}}\right)_{2}\left(\mathrm{OPr}^{\mathrm{i}}\right)_{6}\right]$} \\
\hline Experimental & 674 & 730,470 \\
TDDFT & $678,655,623$ & $701,639,432,412$ \\
(B3LYP/LANL2DZ) & & \\
INDO/S & $767,750,742$ & 820,444 \\
\hline
\end{tabular}

* Geometria otimizada no nível HF/6-31G

A comparação dos espectros experimentais de absorção no infravermelho, obtidos tanto para o sólido cristalino quanto para a solução em baixa, média e alta temperaturas (Figura 4), com os espectros calculados para as espécies $\left[\mathrm{V}\left(\mathrm{OPr}^{\mathrm{i}}\right)_{4}\right]$ e $\left[\mathrm{V}_{2}\left(\mu-\mathrm{OPr}^{\mathrm{i}}\right)_{2}\left(\mathrm{OPr}^{\mathrm{i}}\right)_{6}\right]$ (Tabela 2) também confirma a hipótese proposta como explicação do efeito termocrômico. A diminuição gradativa da intensidade dos sinais espectroscópicos em 720 (este mascarado pelo sinal intenso, em $724 \mathrm{~cm}^{-1}$, do hexano), 640, 613, 583 e $512 \mathrm{~cm}^{-1}$ - característicos da espécie dimérica - com o aumento da temperatura e sem a substituição por sinais característicos da espécie monomérica é indicativa de que o comportamento termocrômico possa ser entendido com base no equilíbrio de dimerização, no qual a espécie dimérica predomina no estado sólido e em baixas temperaturas, transformando-se na espécie monomérica em temperaturas superiores. Ambas as metodologias HF e DFT conseguem prever com razoável acurácia a posição dos sinais na espectroscopia vibracional, com erros geralmente inferiores a $5 \%$.

Para o sistema $\left[\mathrm{V}\left(\mathrm{OPr}^{\mathrm{i}}\right)_{4}\right] /\left[\mathrm{V}_{2}\left(\mu-\mathrm{OPr}^{\mathrm{i}}\right)_{2}\left(\mathrm{OPr}^{\mathrm{i}}\right)_{6}\right]$, o valor experimental do $\Delta \mathrm{H}$ de dimerização, $-54,6 \mathrm{~kJ} \mathrm{~mol}^{-1}$, foi calculado a partir das constantes de equilíbrio determinadas em função da temperatura. ${ }^{6}$ Este valor também é compatível com um processo de agregação molecular em que a formação das pontes $\mu-\mathrm{OPr}^{\mathrm{i}}$ contribui para o abaixamento da energia livre do sistema.

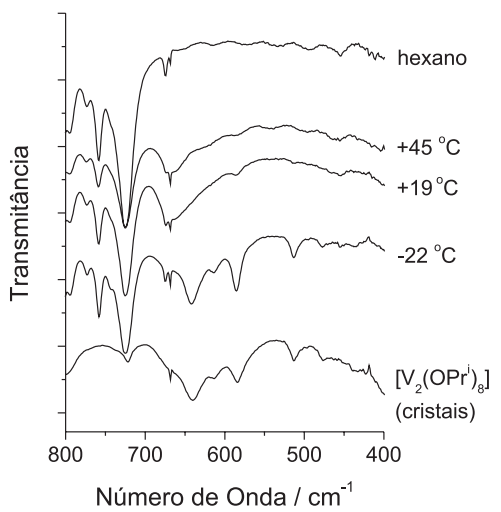

Figura 4. Espectros $I V-T F$ do hexano (solvente), do $\left[\mathrm{V}_{2}\left(\mu-\mathrm{OPr}^{i}\right)_{2}\left(\mathrm{OPr}^{i}\right)_{6}\right]$ sólido (cristalino) e de suas soluções a $-22,+19 e+45^{\circ} \mathrm{C}$

\section{Sistema $\left[\mathrm{V}_{2}(\mu-\mathrm{ONep})_{2}(\mathrm{ONep})_{6}\right] /\left[\mathrm{V}(\mathrm{ONep})_{4}\right]$}

De acordo com evidências oriundas da espectroscopia paramagnética eletrônica (RPE), a multiplicidade de spin $(2 \mathrm{~S}+1)$ da forma dimérica $\left[\mathrm{V}_{2}(\mu-\mathrm{ONep})_{2}(\mathrm{ONep})_{6}\right]$ corresponde a um estado fundamental tripleto. Esta informação, juntamente com a geometria molecular determinada por difratometria de raios $\mathrm{X}$, formaram o ponto de partida para os cálculos relativos à espécie binuclear. Para o monômero, [V(ONep) $\left.{ }_{4}\right]$, uma estrutura gerada a partir de distâncias e ângulos de ligação médios relatados para outros compostos de vanádio e uma multiplicidade de spin do estado fundamental igual a 2 foram as premissas adotadas no início do cálculo.

Também neste caso, como no complexo com isopropóxido, a geometria experimental da espécie dimérica foi mantida, nos cálculos, com uma precisão de cinco casas decimais para distâncias de ligação, e de três casas para ângulos de ligação. Já o [V(ONep) $)_{4}$ otimizado apresentou uma geometria tetraédrica praticamente perfeita, com um ângulo de ligação O-V-O médio de $109,1(8)^{\circ}$ e uma distância de ligação V-O média de 1,7824(3) ̊. A ausência de distorção, neste caso, pode ser explicada pela presença, no neopentóxido, de um grupo "espaçador" - $\mathrm{CH}_{2}-$ que não existe no isopropóxido e que afasta o metal da porção mais volumosa da cadeia carbônica do ligante. Em consequência disso, a repulsão intramolecular diminui e o centro de vanádio(IV) assume uma geometria de coordenação mais regular.

Soluções de $\left[\mathrm{V}_{2}(\mu \text {-ONep })_{2}(\mathrm{ONep})_{6}\right]$ (Figura 2) em tolueno apresentam, da mesma forma que soluções do complexo análogo $\left[\mathrm{V}_{2}\left(\mu-\mathrm{OPr}^{\mathrm{i}}\right)_{2}\left(\mathrm{OPr}^{\mathrm{i}}\right)_{6}\right]$, uma variação espectral significativa com a mudança de temperatura (compare as Figuras 3 e 5). Neste caso são observados também dois padrões nos espectros: em altas temperaturas, há uma banda com máximo em $665 \mathrm{~nm}$, que diminui de intensidade com o abaixamento da temperatura, ao passo que uma banda alargada em torno de $478 \mathrm{~nm}$ se faz mais pronunciada, sendo acompanhada por um crescimento na intensidade de absorção em torno de $730 \mathrm{~nm}$. Devido à semelhança estrutural e dos espectros eletrônicos, o comportamento termocrômico observado para este neopentóxido de vanádio(IV) também foi atribuído, inicialmente, a um equilíbrio de agregação monômero/dímero.

As espécies $\left[\mathrm{V}(\mathrm{ONep})_{4}\right]$ e $\left[\mathrm{V}_{2}(\mu \text {-ONep })_{2}(\mathrm{ONep})_{6}\right]$ tiveram os seus espectros eletrônicos simulados. Para o $\left[\mathrm{V}(\mathrm{ONep})_{4}\right]$, os resultados foram semelhantes aos já apresentados para o $\left[\mathrm{V}\left(\mathrm{OPr}^{\mathrm{i}}\right)_{4}\right]$. Assim, a transição experimental que ocorre com máximo em $665 \mathrm{~nm}$ foi calculada pelos métodos TDDFT e INDO/S como ocorrendo em 616 e 681 $\mathrm{nm}$, respectivamente. Para a espécie $\left[\mathrm{V}_{2}(\mu-\mathrm{ONep})_{2}(\mathrm{ONep})_{6}\right]$, por sua vez, as transições foram calculadas pelo método TDDFT em 397, 611 e $778 \mathrm{~nm}$. Para ambas as espécies há também uma absorção na região do infravermelho, com força de oscilador pequena, que foi atribuída a uma transição eletrônica do orbital HOMO para o LUMO + 1, ambos baseados em orbitais atômicos $d$ do metal. As demais transições entre 
Tabela 2. Principais sinais nos espectros de IV-TF experimentais para o $\left[\mathrm{V}_{2}\left(\mu-\mathrm{OPr}^{\mathrm{i}}\right)_{2}\left(\mathrm{OPr}^{\mathrm{i}}\right)_{6}\right]$ cristalino e as suas soluções em baixa e altas temperaturas, assim como nos espectros simulados para $\left[\mathrm{V}_{2}\left(\mu-\mathrm{OPr}^{\mathrm{i}}\right)_{2}\left(\mathrm{OPr}^{\mathrm{i}}\right)_{6}\right]$ e $\left[\mathrm{V}\left(\mathrm{OPr}^{\mathrm{i}}\right)_{4}\right]$, pelos métodos B3LYP/LANL2DZ e HF/6-31G

\begin{tabular}{|c|c|c|c|c|c|c|}
\hline \multirow{2}{*}{$\begin{array}{l}\text { Espectros Experimentais } \\
\text { Números de Onda } / \mathrm{cm}^{-1} \\
\text { Cristal }\left[\mathrm{V}_{2}\left(\mu-\mathrm{OPr}^{\mathrm{i}}\right)_{2}\left(\mathrm{OPr}^{\mathrm{i}}\right)_{6}\right]\end{array}$} & \multirow[b]{2}{*}{$\begin{array}{l}\text { Solução } \\
-22^{\circ} \mathrm{C}\end{array}$} & \multicolumn{2}{|c|}{$\begin{array}{c}\text { Calculado } \\
{\left[\mathrm{V}_{2}\left(\mu-\mathrm{OPr}^{\mathrm{i}}\right)_{2}\left(\mathrm{OPr}^{\mathrm{i}}\right)_{6}\right] / \mathrm{cm}^{-1}}\end{array}$} & \multirow{2}{*}{$\begin{array}{c}\text { Espectro Experimental } \\
\text { Números de Onda } / \mathrm{cm}^{-1} \\
\begin{array}{c}\text { Solução } \\
+45^{\circ} \mathrm{C}\end{array}\end{array}$} & \multicolumn{2}{|c|}{$\begin{array}{c}\text { Calculado } \\
{\left[\mathrm{V}\left(\mathrm{OPr}^{\mathrm{i}}\right)_{4}\right] / \mathrm{cm}^{-1}}\end{array}$} \\
\hline & & $\mathrm{HF} / 6-31 \mathrm{G}$ & $\begin{array}{c}\text { B3LYP/ } \\
\text { LANL2DZ }\end{array}$ & & $\begin{array}{c}\text { B3LYP/ } \\
\text { LANL2DZ }\end{array}$ & $\mathrm{HF} / 6-31 \mathrm{G}$ \\
\hline 512 & 513 & $491 / 508$ & 498 & - & - & - \\
\hline 584 & 586 & 560 & 560 & - & - & - \\
\hline 613 & 613 & 579 & 578 & - & - & - \\
\hline 640 & 642 & 691 & 604 & - & - & - \\
\hline 804 & 800 & 808 & 791 & - & - & - \\
\hline 823 & 822 & 821 & 817 & - & - & - \\
\hline 850 & 852 & 837 & 832 & 847 & 830 & 840 \\
\hline 928 & 930 & 940 & 898 & - & - & - \\
\hline 938 & 937 & 995 & 924 & 965 & 931 & 1005 \\
\hline 966 & 980 & 1011 & 957 & 996 & 949 & - \\
\hline 996 & 1000 & 1043 & 978 & - & - & - \\
\hline 1017 & - & - & - & - & - & - \\
\hline- & - & 1146 & - & - & - & - \\
\hline 1110 & 1111 & 1160 & 1103 & 1112 & 1093 & 1018 \\
\hline 1127 & 1136 & 1178 & 1129 & 1130 & $1118 / 1127$ & 1143 \\
\hline 1162 & 1163 & 1195 & 1160 & 1165 & 1160 & 1198 \\
\hline 1260 & 1259 & 1366 & 1288 & 1247 & 1305 & 1359 \\
\hline 1323 & 1345 & 1366 & 1305 & - & - & - \\
\hline
\end{tabular}

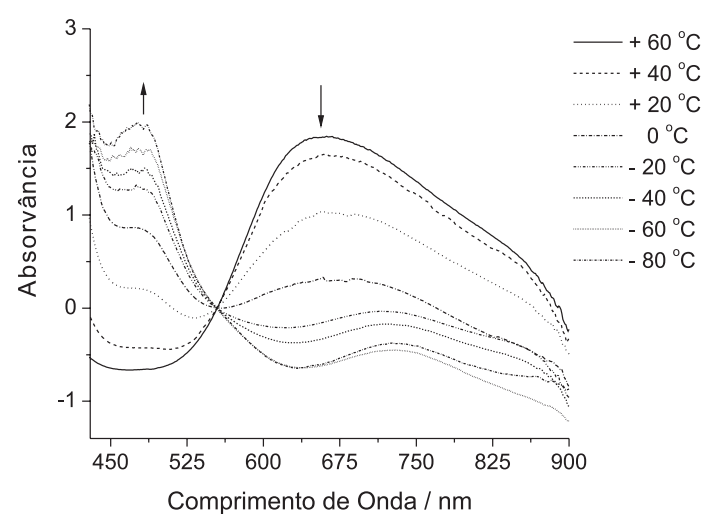

Figura 5. Espectro eletrônico da solução de $\left[\mathrm{V}_{2}(\mu-\mathrm{ONep})_{2}(\mathrm{ONep})_{6}\right]$, concentração $1,84 \times 10^{-2} \mathrm{~mol} \mathrm{~L} \mathrm{~L}^{-1}$ em tolueno, em temperaturas de $-80 \mathrm{a}+60{ }^{\circ} \mathrm{C}$. As setas indicam o sentido da variação de absorvância em resposta à diminuição da temperatura

orbitais de fronteira não apresentaram força de oscilador significativa.

A análise dos resultados revela claramente que o espectro eletrônico calculado para a espécie $\left[\mathrm{V}_{2}(\mu-\mathrm{ONep})_{2}(\mathrm{ONep})_{6}\right]$ difere do espectro experimental obtido em baixas temperaturas: a absorção que deveria ser observada em $478 \mathrm{~nm}$ não aparece como resultado dos cálculos. Além disto, os sinais calculados para $\left[\mathrm{V}_{2}(\mu-\mathrm{ONep})_{2}(\mathrm{ONep})_{6}\right]$ são aqueles que experimentalmente decrescem com a diminuição da temperatura, quando um comportamento contrário seria o esperado. A hipótese de que a forma dimérica não participa do mecanismo que origina o termocromismo é também sugerida pelo fato de que os espectros vibracionais registrados em solução (Figura 6) não são similares ao espectro do $\left[\mathrm{V}_{2}(\mu-\mathrm{ONep})_{2}(\mathrm{ONep})_{6}\right]$ sólido (cristalino), pelo menos nas temperaturas investigadas experimentalmente. Neste contexto, uma explicação alternativa para o efeito termocrômico residiria num equilíbrio entre a forma monomérica e outra de maior grau de agregação, diferente do dímero. O menor grau de agregação superior ao dímero, compatível com a fórmula mínima dos alcóxidos moleculares de vanádio(IV) é o do tetrâmero $\left[\mathrm{V}_{4}(\mu-\mathrm{ONep})_{6}(\mathrm{ONep})_{10}\right]$, representado tentativamente na Figura 7. Na estrutura do tetrâmero pode-se observar que há centros metálicos em dois ambientes químicos diferentes, ligados a cinco ou seis ligantes neopentóxidos em geometria bipiramidal (base triangular) ou octaédrica distorcidas, respectivamente. A estrutura tetramérica mostrada na Figura 7 foi obtida através da duplicação da estrutura dimérica otimizada, preservando-se ao máximo as distâncias e ângulos de ligação.

Para a espécie tetramérica, as transições foram calculadas pelo método INDO/S, considerando um quinteto $(2 \mathrm{~S}+1=5)$ como o estado fundamental, sendo que os principais sinais calculados se localizam em 428 (extremamente intenso), 499, 523, 775 e 783 nm (intensidades baixas). A análise destes resultados sugere que, se assumirmos que a banda experimental alargada em torno de $730 \mathrm{~nm}$ (Figura 5) decorre da sobreposição dos sinais calculados em 775 e 783 nm, e que o mesmo vale para os sinais em 499 e $523 \mathrm{~nm}$ em relação ao ombro experimental em $478 \mathrm{~nm}$, a hipótese de uma espécie tetramérica predominante em baixas temperaturas poderá ser considerada consistente.

A análise dos espectros de absorção no infravermelho (Figura 6) permite mostrar que há boa concordância entre a curva experimental para o $\left[\mathrm{V}_{2}(\mu \text {-ONep })_{2}(\mathrm{ONep})_{6}\right]$ cristalino e a curva calculada para a espécie dimérica. Porém, a concordância entre os pares (curvas simu- 


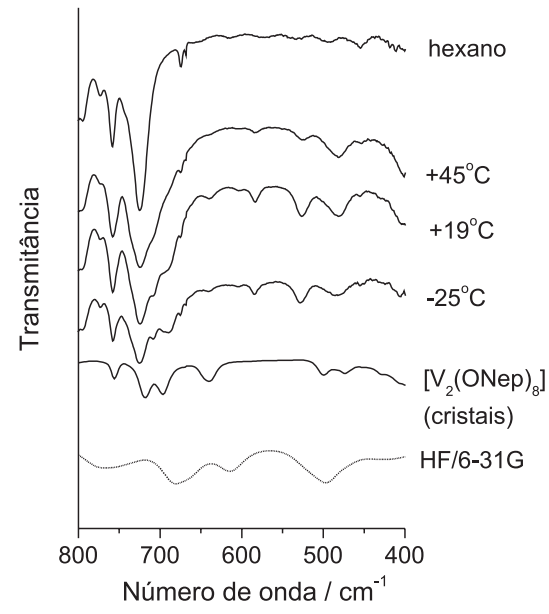

Figura 6. Espectros IV-TF do hexano (solvente), do $\left[V_{2}\left(\mu\right.\right.$-ONep) $\left.{ }_{2}(\mathrm{ONep})_{6}\right]$ sólido (cristalino) e de suas soluções a $-25,+19 e+45^{\circ} \mathrm{C}$, juntamente com o espectro da forma dimérica simulada por $\mathrm{HF} / 6-31 \mathrm{G}$

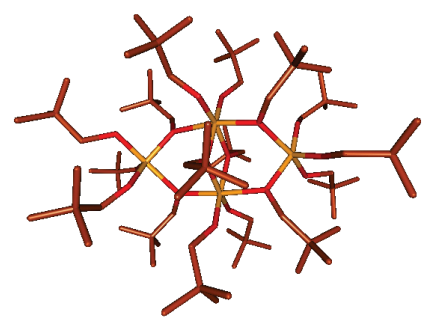

Figura 7.Representação estrutural tentativa da espécie tetranuclear $\left[V_{4}(\mu \text {-ONep })_{6}(\mathrm{ONep})_{10}\right]$, gerada pela duplicação da estrutura dimérica otimizada

ladas para as espécies monomérica e tetramérica e as suas possíveis congêneres experimentais, curvas a $+45^{\circ} \mathrm{C}$ e a $-25^{\circ} \mathrm{C}$, respectivamente) foi insuficiente para que se pudesse afirmar conclusivamente que o termocromismo neste sistema opera segundo um mecanismo de oligomerização entre a forma monomérica e a tetramérica. Em resumo, com base nas diferenças entre os espectros experimentais e calculados de absorção no infravermelho, é possível afirmar que as espécies ou formas de agregação presentes em solução, em baixas temperaturas, não são as mesmas do estado cristalino.

\section{CONCLUSÕES}

O comportamento termocrômico das espécies $\left[\mathrm{V}_{2}\left(\mu-\mathrm{OPr}^{\mathrm{i}}\right)_{2}\left(\mathrm{OPr}^{\mathrm{i}}\right)_{6}\right]$ e $\left[\mathrm{V}_{2}(\mu-\mathrm{ONep})_{2}(\mathrm{ONep})_{6}\right]$ em solução foi estudado por espectroscopia eletrônica, espectroscopia vibracional e técnicas de modelagem molecular. Os resultados indicam claramente que, nas soluções de $\left[\mathrm{V}_{2}\left(\mu-\mathrm{OPr}^{\mathrm{i}}\right)_{2}\left(\mathrm{OPr}^{\mathrm{i}}\right)_{6}\right]$, o termocromismo se origina de um equilíbrio de dimerização, enquanto nas soluções de $\left[\mathrm{V}_{2}(\mu-\mathrm{ONep})_{2}(\mathrm{ONep})_{6}\right]$ os indícios são para um equilíbrio de tetramerização. Entretanto, a precária concordância entre os espectros de absorção no infravermelho calculados para as espécies $\left[\mathrm{V}(\mathrm{ONep})_{4}\right]$ e $\left[\mathrm{V}_{4}(\mu \text {-ONep })_{6}(\mathrm{ONep})_{10}\right] \mathrm{e}$ os espectros experimentais registrados em alta e baixa temperaturas, respectivamente, não permite afirmar com segurança que a forma tetramérica seja majoritária quando o sistema é submetido ao resfriamento.

\section{AGRADECIMENTOS}

A. C. de Freitas, K. C. M. Westrup e G. G. Nunes agradecem ao CNPq pelas bolsas de estudos (mestrado, iniciação científica e pósdoutorado). E. L. de Sá e J. F. Soares agradecem ao CNPq (projetos
200413/2005-8 e 476715/2008-3), à FINEP (CT-INFRA) e ao Laboratório Central de Processamento de Alto Desempenho (LCPAD/UFPR) pelo apoio financeiro e pela infra-estrutura para a realização deste trabalho.

\section{REFERÊNCIAS}

1. Kessler, V. G.; Chem. Commun. 2003, 1213.

2. Hubert-Pfalzgraf, L. G.; Inorg. Chem. 2003, 6, 102.

3. Gulliver, E. A.; Garvey, J. W.; Wark, T. A.; Hampden-Smith, M. J.; Datye, A.; J. Am. Ceram. Soc. 2005, 74, 1091; Barnhart, D. M.; Clark, D. L.; Gordon, J. C.; Huffman, J. C.; Watkin, J. G.; Zwick, B. D.; Inorg. Chem. 1995, 34, 5416.

4. Bradley, D. C.; Mehta, M. L.; Can. J. Chem. 1962, 40, 1183.

5. Kempe, R.; Spannenberg, A.; Z. Kristallogr. - New Cryst. Struct. 1997, $212,485$.

6. Nunes, G. G.; Friedermann, G. R.; dos Santos, J. L. B.; Herbst, M. H.; Vugman, N. V.; Hitchcock, P. B.; Leigh, G. J.; Sá, E. L.; da Cunha, C. J.; Soares, J. F.; Inorg. Chem. Commun. 2005, 8, 83.

7. Nunes, G. G.; Tese de Doutorado, Universidade Federal do Paraná, Brasil, 2004.

8. Reis, D. M.; Westrup, K. C. M.; Nunes, G. G.; Barison, A.; Ribeiro, R. R.; Sá, E. L. de; Hughes, D. L.; Soares, J. F.; J. Braz. Chem. Soc. 2009 , 20, 613; Westrup, K. C. M.; Reis, D. M.; Nunes, G. G.; Barison, A.; Ribeiro, R. R.; Sá, E. L.; Hitchcock, P. B.; Soares, J. F.; Cambridge Structural Database, 2008, deposition number CCDC 705491.

9. Perrin, D. D.; Armarego, W. L. F.; Purification of Laboratory Chemicals, $3^{\text {rd }}$ ed., Butterworth Heenemann: Oxford, 1997.

10. Tomasi, J.; Persico, M.; Chem. Rev. 1994, 94, 2027.

11. Ditchfield, R.; Hehre, W. J.; Pople, J. A.; J. Chem. Phys. 1971, 54, 724.

12. Schmidt, M. W.; Baldridge, K. K.; Boatz, J. A.; Elbert, S. T.; Gordon, M. S.; Jensen, J. H.; Koseki, S.; Matsunaga, N.; Nguyen, K. A.; Su, S.; Windus, T. L.; Dupuis, M.; Montgomery, J. A.; J. Comput. Chem. 1993, $14,1347$.

13. Hay, P. J.; Wadt, W. R.; J. Chem. Phys. 1985, 82, 270.

14. Gaussian 03, Revision D.01, Frisch, M. J.; Trucks, G. W.; Schlegel, H. B.; Scuseria, G. E.; Robb, M. A.; Cheeseman, J. R.; Montgomery, Jr., J. A.; Vreven, T.; Kudin, K. N.; Burant, J. C.; Millam, J. M.; Iyengar, S. S.; Tomasi, J.; Barone, V.; Mennucci, B.; Cossi, M.; Scalmani, G.; Rega, N.; Petersson, G. A.; Nakatsuji, H.; Hada, M.; Ehara, M.; Toyota, K.; Fukuda, R.; Hasegawa, J.; Ishida, M.; Nakajima, T.; Honda, Y.; Kitao, O.; Nakai, H.; Klene, M.; Li, X.; Knox, J. E.; Hratchian, H. P.; Cross, J. B.; Bakken, V.; Adamo, C.; Jaramillo, J.; Gomperts, R.; Stratmann, R. E.; Yazyev, O.; Austin, A. J.; Cammi, R.; Pomelli, C.; Ochterski, J. W.; Ayala, P. Y.; Morokuma, K.; Voth, G. A.; Salvador, P.; Dannenberg, J. J.; Zakrzewski, V. G.; Dapprich, S.; Daniels, A. D.; Strain, M. C.; Farkas, O.; Malick, D. K.; Rabuck, A. D.; Raghavachari, K.; Foresman, J. B.; Ortiz, J. V.; Cui, Q.; Baboul, A. G.; Clifford, S.; Cioslowski, J.; Stefanov, B. B.; Liu, G.; Liashenko, A.; Piskorz, P.; Komaromi, I.; Martin, R. L.; Fox, D. J.; Keith, T.; Al-Laham, M. A.; Peng, C. Y.; Nanayakkara, A.; Challacombe, M.; Gill, P. M. W.; Johnson, B.; Chen, W.; Wong, M. W.; Gonzalez, C.; Pople, J. A.; Gaussian, Inc., Wallingford CT, 2004.

15. Stratmann, R. E.; Scuseria, G. E.; Frisch, M. J.; J. Chem. Phys. 1998 , 109, 8218; Bauernschmitt, R.; Ahlrichs, R.; Chem. Phys. Lett. 1996, 256, 454; Casida, M. E.; Jamorski, C.; Casida, K. C.; Salahub, D. R.; J. Chem. Phys. 1998, 108, 4439.

16. Ridley, J. E.; Zerner, M. C.; Theor. Chim. Acta 1973, 32, 111; Ridley, J. E.; Zerner, M. C.; Theor. Chim. Acta 1976, 42, 223; Bacon, A. D.; Zerner, M. C.; Theor. Chim. Acta 1979, 53, 21.

17. Nemykin, V. N.; Hadt, R. G.; Belosludov, R. V.; Mizuseki, H.; Kawazoe, Y.; J. Phys. Chem. A 2007, 111, 12901.

18. Haaland, A.; Rypdal, K.; Volden, H. V.; Andersen, R. A.; J. Chem. Soc., Dalton Trans. 1992, 891. 